\title{
Biodiversity - economy or ecology? Long-term study of changes in the biodiversity of aphids living in steppe-like grasslands in Central Europe
}

\author{
Barbara OSIADACZ ${ }^{1}$, Roman HAŁAJ ${ }^{2}$ and Damian CHMURA ${ }^{3}$ \\ ${ }^{1}$ Department of Entomology and Environmental Protection, Poznań University of Life Sciences, Dąbrowskiego St. 159, \\ PL 60-594 Poznań, Poland; e-mail: osiadacz@up.poznan.pl \\ ${ }^{2}$ The Upper Silesian Nature Society, Huberta St. 35, PL 40-543 Katowice, Poland; e-mail: roman-halaj@hotmail.com \\ ${ }^{3}$ Institute of Environmental Protection and Engineering, University of Bielsko-Biała, Willowa 2, PL 43-309 Bielsko-Biała, Poland; \\ e-mail: dchmura@ath.bielsko.pl
}

Key words. Hemiptera, Aphidoidea, bio-ecological groups, community structure, protected habitats, loss of biodiversity, human impact, NMDS methods, regional hotspots

\begin{abstract}
This paper examines the changes in the species composition of aphids living in dry calcareous grasslands in Central Europe over a 25 -year period. To the best of our knowledge, this is the first analysis of this type in the world that takes into account both previous and current data on species richness as well as groups of aphids that are distinguishable on the basis of biological and ecological criteria such as host-alternation and feeding types, life cycle, ecological niche, symbiosis with ants and their ecological functional groups. Over the period of more than 25 years, there has been a significant decrease in aphid $\alpha$-diversity, from 171 to 105 species. The gain, which is in species not previously recorded, was 17 taxa. The loss of biodiversity occurred despite the fact that these habitats are protected and are valuable regional biodiversity hotspots. The losses are mostly related to intensive human activity in adjacent areas, which, unfortunately, has resulted in the isolation of these small, protected environmental islands by the removal of ecological corridors. Since, as is shown in this study, the frequencies between individual biological and ecological groups of aphids have been retained, it would be possible to restrict this loss of biodiversity if appropriate actions are taken.
\end{abstract}

\section{INTRODUCTION}

The fragments of steppe-like grasslands that still occur in Central Europe are characterized by a high richness of plant and animal species, especially small arthropods. This is why they are also called "regional biodiversity hotspots" (Cremene et al., 2005) and are protected as nature reserves (CRFOP, 2010) or included in the Natura 2000 European network (European Commission, 2013). Unfortunately, strong human interference in recent decades has caused the majority of these fragments of grassland to become smaller and smaller. At present, they exist only as small isolated "habitat islands", and this may have resulted in biodiversity loss (Burkey, 1989; Collinge, 2000; Krauss et al., 2010).

Therefore, an important question is whether such an unfavourable process really occurs in these protected habitats. It is only possible to answer this question if we have the appropriate comparative data. This is possible for the presented group of insects as we have complete historical data. To fully identify the losses in terms of biodiversity over a period of over 25 years, both the former and current species richness of aphids were analyzed, as well as groups of aphids that were distinguished on the basis of specific biological and ecological criteria. This made it possible to determine trends in the transformation of the aphid communities in these habitats and the losses or gains in terms of the biodiversity of Aphidoidea, which was the main goal of this study.

\section{MATERIAL AND METHODS}

\section{Study areas}

This comparative study was carried out in three steppe nature reserves (A - Krzyżanowice, 50 27'13"N, 20 33'36"E; B - Skowronno, 50 $32^{\prime} 32.58^{\prime \prime} \mathrm{N}, 20^{\circ} 29^{\prime} 10.76^{\prime \prime} \mathrm{E}$; C - Winiary Zagojskie, $50^{\circ} 25^{\prime} 52^{\prime \prime} \mathrm{N}, 20^{\circ} 39^{\prime} 43^{\prime \prime} \mathrm{E}$ ), all of which are situated in lowland in the Nida Basin in southern Poland and two sites that are included in the NATURA 2000 programme (D - Góra

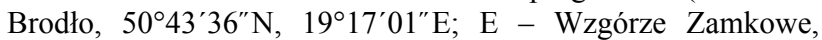
$\left(50^{\circ} 44^{\prime} 58^{\prime \prime} \mathrm{N}, 19^{\circ} 16^{\prime} 26^{\prime \prime} \mathrm{E}\right)$ in the Częstochowa Upland in southern Poland.

All of these sites are of the same phytosociological type, dry calcareous grassland assigned to the Festuco-Brometea class.

\section{Biodiversity data \\ Historical data}

Nida Basin; the data are from the 1980s (Szelegiewicz, 1981; Czylok \& Wojciechowski, 1987), which were supplemented at the beginning of the 1990s (Hałaj, 1996a, b). 
Częstochowa Upland; the complete material comes from the years 1988-1990 (Hałaj, 1996c; Hałaj \& Wojciechowski, 1996).

\section{Current data}

Material from all five sites was collected in 2011-2012 (Osiadacz \& Hałaj, 2015) and supplemented by the authors in 20152016.

\section{Insects collected}

In all cases (in both the historical and present study), aphids were collected using standard methods (by visual inspection of all host plants in an area of approx. $50 \mathrm{~m}^{2}$, at approximately twoweek intervals during the entire vegetative seasons); next, the aphids were prepared (Hałaj \& Osiadacz, 2016) and identified using specialist keys, a list of which is presented in the paper by Blackman \& Eastop (2016). Historical specimens are deposited in the collection of the Museum and Institute of Zoology of Polish Academy of Sciences in Warsaw and the Department of Zoology of the University of Silesia in Katowice, while more recently collected specimens are deposited in the collection of the Department of Entomology and Environmental Protection of Poznan University of Life Sciences.

\section{Analyses}

\section{Biological and ecological criteria}

In addition to the strictly qualitative comparisons (species composition), the aphids were also compared in terms of biological and ecological criteria.

Host-feeding types (Heie, 1980; Hałaj \& Osiadacz, 2016):

FT- $\alpha$ (monophagous and narrow oligophagous), species with a narrow host range that are trophically connected with one species or one genus of host plants;

FT- $\beta$ (oligophagous and polyphagous), species with a broad host range, which includes the remaining aphids.

Life cycle (Heie, 1980; Blackman \& Eastop, 2016; Hałaj \& Osiadacz, 2016):

LC- $\alpha$ (anholocyclic), aphids with an incomplete life cycle, which reproduce only by parthenogenesis;

LC- $\beta$ (holocyclic), aphids with a complete life cycle with a sexual generation and overwintering fertilized eggs.

Host-alternating (Heie, 1980; Blackman \& Eastop, 2016; Hałaj \& Osiadacz, 2016):

HA- $\alpha$ (monoecious), aphids that do not change their host during their life cycle;

HA- $\beta$ (heteroecious), aphids that change their host during their life cycle.

Ecological niche (Blackman \& Eastop, 2016):

EN- $\alpha$ : aphids that feed on the aboveground parts of plants, i.e. stalks, leaves, inflorescences;

EN- $\beta$ : aphids that feed on ground level and underground parts of plants, i.e. root collars and roots.

Symbiosis type (Blackman \& Eastop, 2016; Depa \& Wojciechowski, 2008):

SA- $\alpha$ : aphids obligatorily attended by ants;

SA- $\beta$ : aphids that are not attended by ants or only rarely.

Ecological functional groups (Osiadacz \& Hałaj, 2016):

EFG- $\alpha$ : specialized species (S) that are monophagous and narrowly oligophagous (FT- $\alpha$ ), mainly monoecious (HA- $\alpha$ ) and holocyclic (LC- $\beta$ ) species of aphids living in forest-free areas covered by a dense growth of grass and herbaceous plants;
EFG- $\beta$ : generalist opportunists $(\mathrm{G})$ that are polyphagous and oligophagous (FT- $\beta$ ), mainly heteroecious (HA- $\beta$ ) and holocyclic or anholocyclic (LC- $\alpha$ or LC- $\beta$ ) species of aphids.

Statistical analysis

Since the data was binary, we applied an indirect nonlinear ordination method, i.e. non-metric multi-dimensional scaling (NMDS), to determine the community composition at the sites studied (Minchin, 1987; Oksanen et al., 2016). Bray-Curtis distance measures were used to construct the dissimilarity matrix.

In order to determine whether there are significant differences in species richness between the historical and current data, a Paired Sample T-test (data was normal, Shapiro-Test: $p>0.05$ ) was used.

In order to determine differences in the frequencies in the functional groups between historical and current data, contingency tables ( $\mathrm{G}$ test) were used.

All statistics were performed using the R Language and Environment ( $\mathrm{R}$ Core Team, 2015, package vegan).

\section{RESULTS}

\section{Losses and gains in species diversity}

The data includes 188 species of aphids that occur in calcareous grasslands (Table 1), which constitutes as much as $12.5 \%$ of the aphid fauna in Europe. Of these, 171 taxa were found previously and only 105 currently. Thus, 66 species have been lost. Losses were also recorded in different regions in this study, a loss of 54 species (from 106 to 52) in the Nida Basin and 38 (from 120 to 82) in the Częstochowa Upland. The greatest increase was recorded at Góra Brodło (site D - as many as 43 species) and the lowest at Krzyżanowice (site A - only 4) (Table 1). During the current research, a slight gain of 17 species was recorded, which were not previously recorded in the calcareous grasslands at these sites. More species were recorded in the Częstochowa Upland (12 - out of which only eight here) and fewer in the Nida Valley (seven - out of which only three here). The largest number of new species (12) was recorded at Wzgórze Zamkowe (site E) and the lowest at Krzyżanowice (site A) and Skowronno (site B), with five species at each site (Table 1).

The losses of species were recorded for all the groups of aphid species distinguished and analyzed. The greatest losses were recorded for the holocyclic species (group LC- $\beta-76$ species) and monoecious species (HA- $\alpha-70)$, which live above ground ( $\mathrm{EN}-\alpha-55)$ and are not attended by ants or only sometimes visited by ants (SA- $\beta-50)$, which, at the same time, are specialists with a narrow host range (SE- $\alpha-46)$ (Table 1).

\section{Changes in species richness and composition}

The average species richness during the course of this study decreased significantly at the sites studied $(\mathrm{t}$ $=-3.8197, \mathrm{p}=0.01878$ ) from $65.6 \pm 23$ to $41.4 \pm 16.2$. The NMDS analysis revealed that these sites varied over time and the gradient in species richness and differences in species compositions changed along the NMDS2 axis (Fig. 1). Considering the functional structure, there were no significant differences in the frequency of the different biological and ecological groups of species in the histori- 
Table 1. Previous and current occurrence of aphids at the sites studied, taking into account biological and ecological groups. ${ }^{\dagger}-$ lost species, ${ }^{*}-$ new species

\begin{tabular}{|c|c|c|c|c|c|c|c|c|c|c|c|}
\hline \multirow[b]{2}{*}{ Aphid species } & \multicolumn{5}{|c|}{ Biodiversity data (-1 - historical; -2 - current) } & \multicolumn{6}{|c|}{ Biological and ecological criteria } \\
\hline & $\begin{array}{l}\text { Krzyża- } \\
\text { nowice }\end{array}$ & $\begin{array}{l}\text { Skow- } \\
\text { ronno }\end{array}$ & $\begin{array}{c}\text { Winiary } \\
\text { Zagojskie }\end{array}$ & $\begin{array}{c}\text { Góra } \\
\text { Brodło }\end{array}$ & $\begin{array}{l}\text { Wzgórze } \\
\text { Zamkowe }\end{array}$ & $\begin{array}{l}\text { Host- } \\
\text { feeding }\end{array}$ & $\begin{array}{l}\text { Life } \\
\text { cycle }\end{array}$ & $\begin{array}{c}\text { Host- } \\
\text { alternating }\end{array}$ & $\begin{array}{l}\text { Ecological } \\
\text { niche }\end{array}$ & $\begin{array}{l}\text { Symbiosis } \\
\text { with ants }\end{array}$ & $\begin{array}{l}\text { Functional } \\
\text { groups }\end{array}$ \\
\hline
\end{tabular}

Acaudinum centaureae (Koch) Acyrthosiphon chelidonii (Kaltenbach) Acyrthosiphon cyparissiae (Koch) ${ }^{\dagger}$ Acyrthosiphon malvae malvae (Mosley) Acyrthosiphon pisum pisum (Harris) Ammiaphis sii (Koch)

Anoecia corni (F.)

Anoecia furcata (Theobald) ${ }^{\dagger}$

Anoecia stipae Mamontova

Anoecia vagans (Koch) ${ }^{\dagger}$

Anoecia zirnitsi Mordvilko

Anuraphis catonii Hille Ris Lambers ${ }^{\dagger}$

Anuraphis subterranea (Walker) ${ }^{\dagger}$

Aphis acetosae L.

Aphis antherici Holman

Aphis austriaca Hille Ris Lambers

Aphis breviseta Holman ${ }^{+}$

Aphis calaminthae (Börner)

Aphis chloris Koch

Aphis confusa Walker

Aphis coronillae Ferrari

Aphis craccae L. *

Aphis craccivora Koch

Aphis eryngiiglomerata Bozhko *

Aphis euphorbiae Kaltenbach

Aphis fabae Scopoli *

Aphis galiiscabri Schrank

Aphis helianthemi Ferrar

Aphis hieracii Schrank ${ }^{\dagger}$

Aphis jacobaeae Schrank

Aphis klimeschi (Börner)

Aphis korshunovi Ivanovskaja

Aphis lambersi (Börner)

Aphis leontodontis (Börner)

Aphis molluginis (Börner)

Aphis nastrutii Kaltenbach

Aphis picridis (Börner)

Aphis pilosellae (Börner)

Aphis plantaginis Goetze

Aphis polygonata Nevsky

Aphis pomi de Geer

Aphis proffti (Börner) ${ }^{\dagger}$
Aphis psammophila Szelegiewicz

Aphis roepkei (Hille Ris Lambers) ${ }^{\dagger}$

Aphis salviae Walker *

Aphis sanguisorbae poterii (Börner)

Aphis schilderi (Börner)

Aphis sedi Kaltenbach

Aphis selini (Börner)

Aphis serpylli Koch

Aphis seseli (Bozhko)

Aphis stachydis Mordvilko

Aphis subnitida (Börner)

Aphis subviridis (Börner)
Aphis tacita Huculak ${ }^{\dagger}$

Aphis taraxacicola (Börner) *

Aphis teucrii (Börner)

Aphis thesi Holman
Aphis thomasi (Börner)

Aphis thomasi (Börner) ${ }^{\dagger}$
Aphis triglochinis Theobald

Aphis triglochinis Theobald

Aphis umbrella (Börner) ${ }^{\dagger}$

Aphis urticata J.F. Gmelin

Aphis verbasci Schrank

Aphis verticillatae (Börner) ${ }^{\dagger}$

Aphis (Bursaphis) holoenotherae Rakauskas

Aphis (Pseudoprotaphis) erigerontis (Holman) ${ }^{\dagger}$

Aspidaphis adjuvans (Walker) *

Atheroides serrulatus Haliday

Aulacorthum knautiae Heie ${ }^{+}$

Aulacorthum solani (Kaltenbach)

Brachycaudus (Acaudus) cardui (L.)

Brachycaudus (A.) linariae Stroyan

Brachycaudus (A.) mordvilkoi Hille Ris Labers

Brachycaudus (A.) populi (del Guercio)

Brachycaudus (Appelia) prunicola (Kaltenbach)

Brachycaudus (A.) tragopogonis (Kaltenbach) *

Brachycaudus (Brachycaudus) helichrysi (Kaltenbach)

Brachycaudus (B.) salicinae Börner

Brachycaudus (Scrophulaphis) mimeuri (Remaudière)

Brachycolus cucubali (Passerini)

Brevicoryne brassicae (L.)

Cavariella aegopodii (Scopoli)

Cavariella theobaldi (Gillette et Bragg)

Chaetosiphella stipae Hille Ris Lambers

Chaetosiphon alpestre alpestre Hille Ris Lambers

Cinara juniperi (de Geer)

Coloradoa achilleae Hille Ris Lambers

Coloradoa artemisiae (del Guercio) ${ }^{\dagger}$

Coloradoa campestrella (Ossiannilsson)

Cryptomyzus alboapicalis (Theobald)

Cryptomyzus galeopsidis galeopsidis (Kaltenbach)

Cryptomyzus leonuri (Bozhko) *

Cryptosiphum brevipilosum Börner ${ }^{+}$

\begin{tabular}{|c|c|c|c|c|c|}
\hline- & - & + & - & + & + \\
\hline - & - & - & - & - & - \\
\hline+ & - & - & - & - & - \\
\hline- & - & - & - & - & - \\
\hline+ & + & + & + & - & + \\
\hline- & + & + & + & - & + \\
\hline - & - & + & - & + & + \\
\hline - & - & - & - & - & - \\
\hline - & + & - & + & + & - \\
\hline - & - & - & - & - & - \\
\hline - & - & - & - & - & - \\
\hline - & - & + & - & + & - \\
\hline+ & - & + & - & - & - \\
\hline - & - & - & - & - & - \\
\hline- & - & - & - & - & - \\
\hline - & - & - & - & + & + \\
\hline - & - & - & - & + & - \\
\hline - & - & - & - & + & - \\
\hline - & + & + & + & + & + \\
\hline - & - & - & + & + & + \\
\hline+ & + & - & - & - & - \\
\hline - & + & - & + & - & + \\
\hline+ & - & + & - & + & - \\
\hline - & - & - & - & - & + \\
\hline+ & - & - & - & + & + \\
\hline - & - & - & + & - & - \\
\hline - & + & - & + & - & - \\
\hline - & - & - & + & + & + \\
\hline - & - & - & - & - & - \\
\hline - & - & + & + & - & - \\
\hline - & + & - & - & + & + \\
\hline - & - & - & - & - & - \\
\hline - & - & - & + & + & - \\
\hline - & - & - & - & - & - \\
\hline - & - & - & - & - & - \\
\hline - & - & - & - & + & - \\
\hline - & - & + & - & + & + \\
\hline - & - & - & - & + & - \\
\hline+ & - & - & - & - & - \\
\hline - & - & - & - & - & - \\
\hline - & - & - & - & - & - \\
\hline+ & - & - & - & + & - \\
\hline - & - & - & - & - & - \\
\hline - & - & - & - & + & - \\
\hline - & + & - & + & - & - \\
\hline - & + & - & + & + & + \\
\hline - & + & + & - & - & - \\
\hline - & - & - & - & + & + \\
\hline - & - & + & - & - & - \\
\hline - & + & - & + & + & + \\
\hline - & - & - & - & + & + \\
\hline - & - & - & - & - & - \\
\hline - & - & - & - & - & - \\
\hline - & - & - & - & - & - \\
\hline - & - & - & - & + & - \\
\hline - & - & - & - & - & - \\
\hline - & - & - & - & - & - \\
\hline - & - & - & - & + & + \\
\hline+ & - & + & - & - & - \\
\hline - & - & - & - & + & - \\
\hline+ & - & - & - & - & - \\
\hline - & + & - & - & - & - \\
\hline - & - & + & - & - & - \\
\hline+ & - & - & - & - & - \\
\hline - & - & - & - & - & - \\
\hline - & - & - & - & - & - \\
\hline - & - & - & - & - & - \\
\hline - & - & - & - & + & - \\
\hline - & - & - & - & - & - \\
\hline - & - & + & - & - & - \\
\hline+ & - & - & - & - & - \\
\hline - & - & - & - & - & - \\
\hline - & - & + & - & + & - \\
\hline - & - & - & - & - & - \\
\hline - & - & + & - & - & - \\
\hline - & - & - & - & - & - \\
\hline - & - & - & - & - & - \\
\hline - & + & + & - & + & + \\
\hline - & - & + & - & + & - \\
\hline - & - & - & - & - & - \\
\hline - & - & - & - & - & - \\
\hline - & - & - & - & - & - \\
\hline - & - & - & - & - & - \\
\hline - & - & - & - & + & - \\
\hline - & - & - & - & + & - \\
\hline - & - & - & - & - & - \\
\hline - & - & - & - & + & - \\
\hline - & - & - & - & - & - \\
\hline - & - & - & - & + & - \\
\hline - & - & - & - & - & - \\
\hline - & + & - & + & - & + \\
\hline - & + & - & - & - & + \\
\hline - & - & - & - & - & - \\
\hline
\end{tabular}


Table 1 (continued).

\begin{tabular}{|c|c|c|c|c|c|c|c|c|c|c|c|}
\hline \multirow{3}{*}{ Aphid species } & \multicolumn{5}{|c|}{ Biodiversity data (-1 - historical; -2 - current) } & \multicolumn{6}{|c|}{ Biological and ecological criteria } \\
\hline & $\begin{array}{l}\text { Krzyża- } \\
\text { nowice }\end{array}$ & $\begin{array}{l}\text { Skow- } \\
\text { ronno }\end{array}$ & $\begin{array}{c}\text { Winiary } \\
\text { Zagojskie }\end{array}$ & $\begin{array}{l}\text { Góra } \\
\text { Brodło }\end{array}$ & $\begin{array}{l}\text { Wzgórze } \\
\text { Zamkowe }\end{array}$ & $\begin{array}{c}\text { Host- } \\
\text { feeding }\end{array}$ & $\begin{array}{l}\text { Life } \\
\text { cycle }\end{array}$ & $\begin{array}{c}\text { Host- } \\
\text { alternating }\end{array}$ & $\begin{array}{l}\text { Ecological } \\
\text { niche }\end{array}$ & $\begin{array}{l}\text { Symbiosis } \\
\text { with ants }\end{array}$ & $\begin{array}{l}\text { Functional } \\
\text { groups }\end{array}$ \\
\hline & $\begin{array}{ll}\text { A-1 } & \text { A-2 }\end{array}$ & $\begin{array}{ll}\text { B-1 } & \text { B-2 }\end{array}$ & C-1 C-2 & $\begin{array}{ll}D-1 & D-2\end{array}$ & E-1 $E-2$ & $\overline{\text { FT- } \alpha \text { FT- } \beta}$ & $C-\alpha \mathrm{LC}$ & $3 \mathrm{HA}-\alpha \mathrm{HA}-\beta$ & $E N-\alpha$ EN- $\beta$ & SA- $\alpha$ SA- $\beta$ & $F G-\alpha$ EFG- $\beta$ \\
\hline
\end{tabular}

Diuraphis (Diuraphis) noxia (Kurdjumov)

Dysaphis oeroselini Szelegiewicz

Dysaphis sorbi (Kaltenbach)

Forda marginata Koch

Forda marginata Koch
Geoica urticularia (Passerini) ${ }^{\dagger}$

Hayhurstia atriplicis (L.) *

Hyadaphias hofmanni (Börner)

Hyadaphias molluginis Börner ${ }^{\dagger}$
Hyadaphias mosana Hille Ris Lambers

Hyadaphias mosana Hille Ris Lam
Hyalopteroides humilis (Walker) ${ }^{\dagger}$

Hyperomyzus (Hyperomyzus) lactucae (L.) *

Hyperomyzus (Neonasonovia) picridis (Börner \& Blunck) ${ }^{\dagger}$

Hyperomyzus (N.) zirnitsi Hille Ris Lambers ${ }^{\dagger}$

Izypha bufo (Walker) ${ }^{\dagger}$

Laingia psammae Theobald

Linosiphon galiophagum (Wimshurst)

Liosomaphis berberidis (Kaltenbach)

Lipaphis erysimi (Kaltenbach)

Lipaphis turitella (Wahlgren) ${ }^{\dagger}$

Longicaudus trirhodus (Walker) ${ }^{\dagger}$

Macrosiphoniella artemisiae (Boyer de Fonscolombe)

Macrosiphoniella dimidiata Börner

Macrosiphoniella millefolii (de Geer)

Macrosiphoniella subaequalis Börner

Macrosiphoniella usquertensis Hille Ris Lambers

Macrosiphoniella (Phalangomyzus) oblonga (Mordvilko)

Macrosiphoniella (Ramitrichophorus) medvedevi Bozh.

Macrosiphum euphorbiae (Thomas)

Macrosiphum rosae (L.)

Maculolachnus submacula (Walker)

Megoura viciae Buckton

Metopolophium festucae (Theobald)

Metopolopphium fasciatum Stroyan

Microlophium carnosum (Buckton)
Myzaphis rosarum (Kaltenbach)

Myzus cerasi ( $\mathrm{F}$.)

Myzus langei (Börner)

Nasonovia compositellae nigra (Hille Ris Lambers) ${ }^{\dagger}$

Nasonovia pilosellae (Börner)

Nasonovia ribisnigri (Mosley)

Nevskya fungifera (Ossiannilsson) ${ }^{\dagger}$

Phorodon humuli (Shrank) ${ }^{\dagger}$

Pleotrichophorus duponti Hille Ris Lambers ${ }^{\dagger}$

Pleotrichophorus glandulosus (Kaltenbach) ${ }^{\dagger}$

Pleotrichophorus persimilis Börner ${ }^{\dagger}$

Protaphis carlinae (Börner)

Protaphis elongata (Nevsky) ${ }^{\dagger}$

Protaphis hartigi (Hille Ris Lambers) ${ }^{\dagger}$

Protrama flavescens (Koch) ${ }^{\dagger}$

Protrama flavescens (Koch) ${ }^{\dagger}$
Protrama longitarsus (Ferrari)

Pseudobrevicoryne erysimi Holman

Rhopalomyzus (Judenkoa) lonicerae (Siebold) *

Rhopalosiphum oxyacanthae (Schrank)

Rhopalosiphum maidis (Fitch)

Rhopalosiphum padi (L.)

Schizaphis graminum (Rondani)

Schizaphis jaroslavi
Semiaphis dauci (F.)

Semiaphis dauci (F.)

Semiaphis pimpinellae (Kaltenbach)

Sipha (Rungsia) elegans del Guercio

Sipha (R.) maydis Passerini

Sipha (Sipha) glyceriae (Kaltenbach)

Sitobion avenae ( $F$.)

Sitobion fragariae (Walker) ${ }^{\dagger}$

Smiela fusca Mordvilko

Staegeriella necopinata (Börner)

Taiwanomyzus alpicola (Hille Ris Lambers)

Therioaphis (Pterocallidium) luteola (Börner)

Therioaphis ( $P$.) trifolii (Monell)

Therioaphis (Therioaphis) ononidis (Kaltenbach) ${ }^{\dagger}$

Tetraneura ulmi (L.)

Titanosiphon artemisiae (Koch)

Toxopterina vandergootii (Börner)

Trama centaureae Börne

Trama troglodytes von Heyden ${ }^{\dagger}$
Uroleucon (Uroleucon) achilleae (Koch)

Uroleucon (U.) cichorii (Koch)

Uroleucon (U.) cirsii (L.)

Uroleucon (U.) grossum (Hille Ris Lambers)

Uroleucon (U.) hypochoeridis (F.)

Uroleucon (U.) leontodontis (Hille Ris Lambers) *

Uroleucon (U.) obscurum (Koch)

Uroleucon (U.) picridis (F.) ${ }^{\dagger}$

Uroleucon (U.) pilosellae (Börner)

Uroleucon (Uromelan) aeneum (Hille Ris Lambers) ${ }^{\dagger}$

Uroleucon (U.) campanulae (Kaltenbach)

Uroleucon (U.) carlinae (Börner)

Uroleucon (U.) ensifoliae (Holman)

Uroleucon (U.) jaceae (L.)

Uroleucon (U.) jaceae henrichi (Börner) ${ }^{\dagger}$

Uroleucon (U.) nigrocampanulae (Theobald)

Uroleucon (U.) taraxaci (Kaltenbach)

Xerobion judenkoi (Szelegiewicz) ${ }^{\dagger}$ 


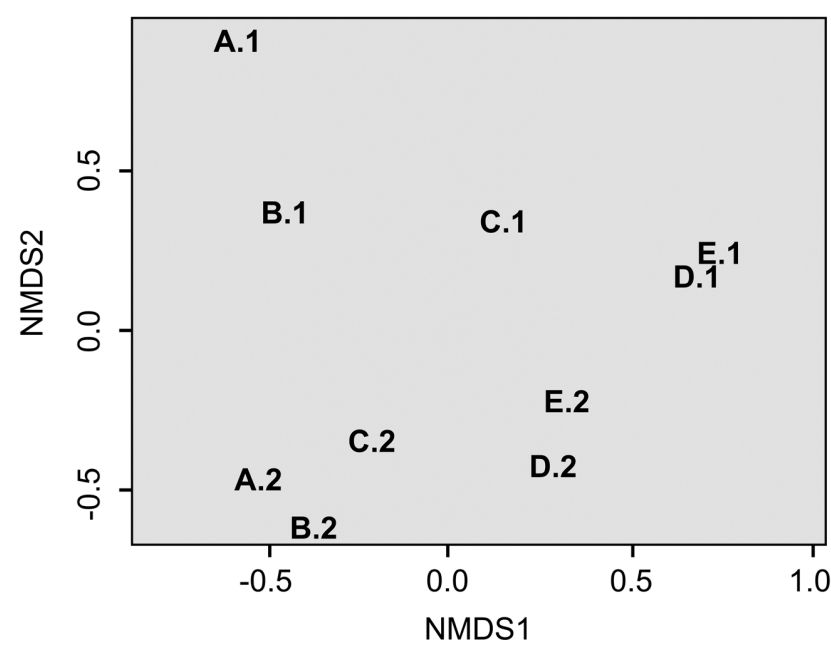

Fig. 1. Non-metric multi-dimensional scaling (NMDS) ordination of the results for all of the sites studied in two-dimensional space. Explanations: A-E - sites; 1 - historical data, 2 - current data.

cal and current records (Table 2). A significant increase at Wzgórze Zamkowe (site E) was recorded in group FT- $\beta$ (oligophagous and polyphagous) compared to that in FT- $\alpha$ (monophagous and narrow oligophagous), and in SE- $\beta$ (generalists) compared to that in SE- $\alpha$ (specialists) (Table 2).

\section{DISCUSSION}

\section{Changes in biodiversity}

Studies in recent years have shown that there have been dramatic decreases in biodiversity (e.g. Butchart et al., 2010; Boyle \& Boyle, 2013; Burns et al., 2014; Kunz, 2016). Our study reveals that this happens not only in well-studied groups of insects, e.g. butterflies, beetles and Hymenoptera etc. (e.g. Stewart et al., 2007; Nowacki \& Wąsala, 2008; Banaszak \& Ratyńska, 2014; Senapathi et al., 2015; Pozsgai et al., 2016), but also in suctorial phytophagous insects, which are rarely thought of in terms of biodiversity. Our analyses have shown a decrease in the $\alpha$-diversity of aphids over a period of more than 25 years at all the sites studied. Although this applies to aphids in all of the distinguished biological and ecological groups, the most worrying is the decrease in the number of specialists (SE- $\alpha$ ) along with a simultaneous increase in the number of generalists (SE- $\beta$ ), which leads to "biotic homogenisation" (Fig. 1). Similar changes are reported in other dry grass- lands studied (Osiadacz \& Hałaj, 2016), which result in the "synanthropisation" of communities. This is most marked at Wzgórze Zamkowe (site E). Many specialist species are no longer recorded there, e.g. those of the genera Aphis and Uroleucon. Coloradoa spp., Pleotrichophorus spp. and Xerobion judenkoi also no longer occur there although their host plant (Artemisia campestris) is as abundant as previously (Urbisz, 2008). This confirms the thesis put forward by Osiadacz \& Hałaj (2015). It is also reported in other types of habitats, e.g. bogs (Hałaj, 1991, 1993) and coniferous and broad-leaved forests (Hałaj \& Wojciechowski, 1997; Durak \& Wojciechowski, 2005) that the presence of host plants is not a sufficient condition for the occurrence of a specific species of aphid. The number of generalists, on the other hand, is associated with the increase in synanthropic plants that colonize these habitats. This also confirms another thesis that changes in the composition of an aphid community are closely related to changes in the composition of plant assemblages (which is also recorded for other invertebrates: Schaffers et al., 2008), which indicate important changes in the habitat (Osiadacz \& Hałaj, 2016). On a much smaller scale, such a phenomenon was recorded at Wzgórze Zamkowe (sites E) and Góra Brodło (site D). As both sites are currently situated in a strongly developing tourist area in the Częstochowa Upland area, it is concluded that the changes in biodiversity are associated with tourism (Hall, 2010). However, despite the fact that a general loss in diversity was recorded at the sites analyzed, the frequencies of the occurrence of aphids in different biological and ecological groups remained the same (except at Wzgórze Zamkowe - site E). This indicates that there have not been similar changes in the habitats there and that it is possible to limit further losses if modern methods of protecting natural resources, which take into account the contemporary achievements of science, were used (Forman, 1995; Kunz, 2016). Therefore, one should consider actively protecting these sites, especially in respect to regaining and restoring ecological corridors. Perhaps, it will be necessary to limit tourism in order to avoid losing these precious dry calcareous grasslands (even the relic ones at Góra Brodło - site D: Wika, 1986).

\section{Changes in landscape}

Due to intensive human activity in a relatively small area, Europe has been and continues to experience a loss of biodiversity. This applies particularly to Central Eu-

Table 2. The percentage of biological and ecological groups of aphids at the sites studied. 1 - historical data, $2-$ current data. Data in bold are significant (G-test) at $\mathrm{p}<0.05$.

\begin{tabular}{|c|c|c|c|c|c|c|c|c|c|c|c|}
\hline Biological and ecological gro & & A-1 & A-2 & B-1 & B-2 & $\mathrm{C}-1$ & $\mathrm{C}-2$ & $\mathrm{D}-1$ & $\mathrm{D}-2$ & $\mathrm{E}-1$ & $\mathrm{E}-2$ \\
\hline \multirow{2}{*}{ Host-feeding types } & FT- $\alpha$ & 35.3 & 50.0 & 45.7 & 38.5 & 59.1 & 56.8 & 51.8 & 47.6 & 54.3 & 30.6 \\
\hline & FT- $\beta$ & 64.7 & 46.7 & 54.3 & 57.7 & 40.9 & 43.2 & 49.4 & 50.0 & 46.7 & 69.4 \\
\hline \multirow{2}{*}{ Life cycle } & LC- $\alpha$ & 2.9 & 10.0 & 6.5 & 11.5 & 12.1 & 10.8 & 8.2 & 4.8 & 8.7 & 5.6 \\
\hline & LC- $\beta$ & 97.1 & 90.0 & 93.5 & 88.5 & 87.9 & 89.2 & 91.8 & 95.2 & 91.3 & 94.4 \\
\hline \multirow{2}{*}{ Host-alternating } & $\mathrm{HA}-\alpha$ & 88.2 & 93.3 & 78.3 & 84.6 & 87.9 & 86.5 & 81.2 & 88.1 & 88.0 & 76.4 \\
\hline & $H A-\beta$ & 11.8 & 6.7 & 21.7 & 15.4 & 12.1 & 13.5 & 18.8 & 11.9 & 12.0 & 23.6 \\
\hline \multirow{2}{*}{ Ecological niche } & $E N-\alpha$ & 82.4 & 80.0 & 65.2 & 84.6 & 65.2 & 70.3 & 72.9 & 83.3 & 73.9 & 83.3 \\
\hline & $E N-\beta$ & 17.6 & 20.0 & 34.8 & 15.4 & 34.8 & 29.7 & 27.1 & 16.7 & 26.1 & 16.7 \\
\hline \multirow{2}{*}{ Symbiosis with ants } & SA- $\alpha$ & 26.5 & 43.3 & 41.3 & 50.0 & 48.5 & 48.6 & 36.5 & 31.0 & 44.6 & 38.9 \\
\hline & $S A-\beta$ & 73.5 & 56.7 & 58.7 & 50.0 & 51.5 & 51.4 & 63.5 & 69.0 & 55.4 & 61.1 \\
\hline \multirow{2}{*}{ Ecological functional groups } & EFG- $\alpha$ & 46.7 & 35.3 & 47.8 & 46.2 & 60.6 & 59.5 & 55.3 & 50.0 & 56.5 & 33.3 \\
\hline & $E F G-\beta$ & 63.3 & 64.7 & 52.2 & 53.8 & 39.4 & 40.5 & 44.7 & 50.0 & 43.5 & 66.7 \\
\hline
\end{tabular}


rope. In this region, the character of the economy changed after changes in the political system. As a result, the direct actions that influence nature have intensified. They are especially visible in the transformation of landscapes, especially by their fragmentation (e.g. new traffic routes, housing, tourist accommodation, agrocenoses, bodies of water etc.). The fragmentation of habitats causes a significant loss of biodiversity (Krauss et al., 2010). This process is significantly influenced by the size of the fragments in the habitats that are saved (if their surface area decreases, this process becomes more intense) or the loss of ecological corridors (no connection between local populations of a species within the metapopulation can lead to its extinction) (Burkey, 1989; Moilanen \& Hanski, 1998; Collinge, 2000; Wallis De Vries et al., 2002). These phenomena are the main causes of the loss of biodiversity in the habitats studied. Although the sites that were analyzed in this study have been protected since the 1950s, due to changes in the political system and the introduction of a market economy, their surrounding area was heavily transformed, which broke the connections between protected patches and sites. In addition, sheep farming, which was undoubtedly a stabilizing factor, was also abandoned (Fischer et al., 1996; Poschlod \& Bonn, 1998). Moreover, the recent intensive development of tourism in these areas is not a factor that promotes the preservation of the status of these areas (Vaughan, 2000).

\section{CONCLUSION}

This analysis demonstrates there has been a considerable decrease in the biodiversity of aphids in the entire area studied over a period of a quarter of a century. This process is especially dangerous because it has resulted in the loss of ubiquitous species, which results in the gradual "homogenisation of communities", which along with a small increase in generalist species leads to the synanthropisation of communities. The main cause of these changes is human activity associated with the economy (development of agriculture and tourism). As a result, the connectivity among local habitat islands no longer exists due to the disappearance of ecological corridors. In addition, such isolated islands gradually become limited territorially (decreased), which reduces the level of connection among the local populations of species within metapopulations, which, along with the decrease in their area, affect the rate of extinction. If we combine these regional factors with global ones (climate change), then the processes of extinction become more dramatic. Obviously, the conflict between "anthropogenization" and biodiversity (since the first human settlements) will continue and its balance will never be equal to zero (as in the natural landscape). However, by applying the principles of sustainable development (taking into account the results of the latest ecological trends), the process can be or even should be mitigated. In the long term, it may turn out that the expected benefits from the infrastructure that is created will only be an insignificant percentage of the losses that are associated with the decrease in biodiversity.

\section{REFERENCES}

BANASZAK J. \& RATYŃSKA H. 2014: Local changes in communities of wild bees (Hymenoptera: Apoidea, Apiformes): 30 years later. — Polish J. Entomol. 83: 325-351.

Blackman R.L. \& Eastop V.F. 2016: Aphids on the World's Plants. An Online Identification and Information Guide. URL: http:// www.aphidsonworldsplants.info (last accessed 4 Sep. 2016).

Boyle T.J.B. \& Boyle C.E.B. 2013: Biodiversity, Temperate Ecosystems, and Global Change. Springer Science \& Business Media, Berlin, Heidelberg, Dordrecht, New York, 456 pp.

BRUUN H.H. 2000: Patterns of species richness in dry grassland patches in an agricultural landscape. _ Ecography 23: 641650 .

BURKEY T.V. 1989: Extinction in nature reserves: the effect of fragmentation and the importance of migration between reserve fragments. - Oikos 55: 75-81.

Burns E., Lindenmayer D., Lowe A. \& Thurgate N. 2014: Biodiversity and Environmental Change: Monitoring, Challenges and Direction. CSIRO, Collingwood, 624 pp.

Butchart S.H.M., Walpole M., Collen B., van Strien A., ScharLemann J.P.W., Almond R.E.M., Baillie J.E.M., Bomhard B., Brown C., Bruno J. ET AL. 2010: Global biodiversity: Indicators of recent declines. - Science 328: 1164-1168.

Collinge S.K. 2000: Effects of grassland fragmentation on insect species loss, colonization, and movement patterns. — Ecology 81: 2211-2226.

Cremene C., Groza G., Rakosy L., Schileyko A.A., Baur A., ErHARDT A. \& BAUR B. 2005: Alterations of steppe-like grasslands in Eastern Europe: a threat to regional biodiversity hotspots. Conserv. Biol. 19: 1606-1618.

CRFOP 2010: Central Register of Forms of Nature Protection. URL: http://crfop.gdos.gov.pl/ (last accessed 4 Sep. 2016).

Czylok A. \& Wojciechowski W. 1987: Communities of aphids (Homoptera, Aphidoidea) of the xerothermic vegetation in Niecka Nidziańska. — Acta Biol. Siles. 6: 37-41.

Depa Ł. \& WoJcIechowsKi W. 2008: Ant-root aphid relations in different plant associations. - Pol. J. Entomol. 77: 151-163.

Durak R. \& Wojciechowski W. 2005: Aphid (Hemiptera: Aphidoidea) communities in different forest associations (VaccinioPiceetes and Querco-Fagetea classes) of the Kolbuszowa Plateau. - Aphids Hemipt. Insects 11: 39-52.

EUROPEAN COMMISSION 2013: Interpretation Manual of European Union Habitats, ver. EUR 28. URL: http://ec.europa.eu/environment/nature/legislation (last accessed 4 Sep. 2016).

Fischer S.F., Poschlod P. \& Beinlich B. 1996: Experimental studies on the dispersal of plants and animals on sheep in calcareous grasslands. - J. Appl. Ecol. 33: 1206-1222.

Forman R.T.T. 1995: Land Mosaics: The Ecology of Landscapes and Regions. Cambridge University Press, Cambridge, 632 pp.

HaŁAJ R. 1991: [The fauna of aphids (Homptera: Aphidoidea) of high peat-bogs "Mszary Izerskie" and "Zieleniec-Topieliska" in the Polish Sudetes.] — Acta Biol. Siles. 18: 126-132 [in Polish, English abstr.].

HaŁaj R. 1993: The fauna of aphids (Homoptera, Aphidinea) of the selected high peat-bogs in the Polish Carpathians. - Acta Biol. Siles. 22: 92-104.

Hataj R. 1996a: Aphids (Sternorrhyncha: Aphidinea) of the "Winiary Zagojskie" natural reserve (Niecka Nidziańska, Małopolska Upland). — Acta Entomol. Siles. 4: 8-10.

HaŁAJ R. 1996b: Aphid species new for Niecka Nidziańska region (Sternorrhyncha: Aphidinea). — Acta Entomol. Siles. 4: 24.

HaŁaj R. 1996c: Five aphid species (Hemiptera: Aphidinea) new to Poland. - Ann. Upper Siles. Mus. Bytom (Entomol.) 6-7: 81-87. 
Hataj R. \& Osiadacz B. 2016: Keys for the Identification of Polish Insects, XVII, 5c. Hemiptera (Homoptera) - Aphidomorpha - Aphidoidea: Hormaphididae, Mindaridae, Phloeomyzidae, Thelaxidae. Polish Entomological Society, Wrocław, 163 pp.

Halaj R. \& Wojciechowski W. 1996: Aphid communities (Homoptera, Aphidinea) connected with Festuco-Brometea and Sedo-Scleranthetea class assotiations (Częstochowska Uplands). — Acta Biol. Siles. 29: 83-105.

Halaj R. \& Wojciechowski W. 1997: Zgrupowania mszyc (Homoptera, Aphidinea) związane z niektórymi zbiorowiskami z klas Vaccinio-Picetea, Alnetea glutinosae i Querco-Fagetea okolic Olsztyna k. Częstochowy. - Acta Biol. Siles. 30: 61-83 [in Polish, English abstr.].

HaLl M.C. 2010: Tourism and biodiversity: more significant than climate change? - J. Herit. Tourism 5: 253-266.

HeIE O.E. 1980: The Aphidoidea (Hemiptera) of Fennoscandia and Denmark. I General part. The families Mindaridae, Hormaphididae, Thelaxidae, Anoeciidae, and Pemphigidae. Fauna Entomologica Scandinavica 9: 236 pp.

Krauss J., Bommarco R., Guardiola M., Heikkinen R.K., Helm A., Kuussaari M., Lindborg R., ÖCkinger E., Pärtel M., Pino J. ET AL. 2010: Habitat fragmentation causes immediate and time-delayed biodiversity loss at different trophic levels. Ecol. Lett. 13: 597-605.

Kunz W. 2016: Species Conservation in Managed Habitats: The Myth of a Pristine Nature. Wiley-VC, Weinheim, 300 pp.

MinCHIN P.R. 1987: An evaluation of relative robustness of techniques for ecological ordinations. - Vegetatio 69: 89-107.

MoIlAnEN A. \& HANSKi I. 1998: Metapopulation dynamics: effects of habitat quality and landscape structure. - Ecology 79 2503-2515.

NOWACKI J. \& WĄSALA R. 2008: Changes in groups of noctuids (Lepidoptera, Noctuidae) of xerothermic rock swards in the Pieniny Mountains, caused by operation of Czorsztyn-Niedzica and Sromowce Wyżne water reservoirs. - Pol. J. Environ. Stud. 17: 71-77.

Oksanen J.F., Blanchet F.G., Friendly M., Kindt R., Legendre P., McGlinn D., Minchin P.R., O'Hara R.B., Simpson G.L., Solymos P., Stevens M.H.H., Szoecs E. \& Wagner H. 2016: The Vegan Package. Community Ecology Package, 10. R Package Ver. 2.4-1. URL: https://CRAN.R-project.org/package=vegan (last accessed 4 Sep. 2016).

Osiadacz B. \& HaŁaj R. 2015: Aphids in jeopardy? Aphid communities on xerothermic habitats. — Biologia 70: 1118-1135.
Osiadacz B. \& HaŁaj R. 2016: Aphids under stress. Species groups and ecological functional groups of aphids define heavy metal grasslands of Central Europe. - Redia 99: 35-51.

Poschlod P. \& BonN S. 1998: Changing dispersal processes in the central European landscape since the last ice age: an explanation for the actual decrease of plant species richness in different habitats? - Acta Bot. Neerl. 47: 27-44.

Pozsgai G., Baird J., Littlewood N., Robin J. Pakeman R.J., MARK R. \& Young M.R. 2016: Long-term changes in ground beetle (Coleoptera: Carabidae) assemblages in Scotland. Ecol. Entomol. 41: 157-167.

R Core Team 2015: R: A Language and Environment for Statistical Computing. R Foundation for Statistical Computing, Vienna.

Schaffers A.P., Raemakers I.P., Sýkora K.V. \& Ter BraAk C.J.F. 2008: Arthropod assemblages are best predicted by plant species composition. - Ecology 89: 782-794.

Senapathi D., Carvalheiro L.G., Biesmeijer J.C., Dodson C.-A., Evans R.L., McKerchar M., Morton R.D., Moss E.D., RobERTS S.P.M., KUNIN W.E. ET AL. 2015: The impact of over 80 years of land cover changes on bee and wasp pollinator communities in England. _ Proc. R. Soc. (B) 282: 20150294, 8 pp.

Stewart A.J.A., New T.R. \& Lewis O.T. 2007: Insect Conservation Biology. Proceedings of the Royal Entomological Society's 23rd Symposium. CABI, Wallingford, $465 \mathrm{pp}$.

SzeLEGIEwICZ H. 1981: Notes on xerothermophilous aphids of the Little Poland Plateau (Homoptera, Aphidoidea). — Fragm. Faun. 25: 423-434.

URBISZ A. 2008: Synopsis of the Vascular Plant Flora of the Kraków-Częstochowa Upland. Prace naukowe Uniwersytetu Śląskiego w Katowicach no. 2630, Wydawnictwo Uniwersytetu Śląskiego, Katowice, 136 pp.

VAUGHAN D. 2000: Tourism and biodiversity: a convergence of interests? - Int. Affairs 76: 283-297.

Wallis DeVries M.F., Poschlod P. \& Willems J.H. 2002: Challenges for conservation of calcareous grasslands in northwestern Europe: integrating the requirements of flora and fauna. Biol. Conserv. 104: 265-273.

WIKA S. 1986: Geobotanical Problems of the Central Part of the Cracow-Wieluń Upland. Prace naukowe Uniwersytetu Śląskiego w Katowicach no. 815, Wydawnictwo Uniwersytetu Śląskiego, Katowice, 156 pp.

Received October 3, 2016; revised and accepted February 8, 2017 Published online March 14, 2017 